\section{Cobertura e adequação do exame citopatológico de colo uterino em estados das regiões Sul e Nordeste do Brasil}

\author{
Pap test coverage and adequacy in the South and \\ Northeast of Brazil
}

\begin{abstract}
Cervical cancer is the second most common cancer among women both in Brazil and elsewhere in the world and can be averted through early detection of precursor lesions. Pap smear is still the most effective and efficient screening test. This study focused on the coverage and adequacy of Pap test and associated factors. The authors adopted a cross-sectional design with a sample of 3,939 women who had given birth in the two previous years in 41 municipalities (counties) of Brazil. Lifetime Pap test coverage was $75.3 \%$ (95\%CI: 74.0-76.7), and prevalence of adequacy was 70.7\% (95\%CI: 69.3-72.1). Adequacy was positively associated with age over 25 years, schooling, prenatal care in the last pregnancy, and gynecological visit in the previous year, and was less frequent among primiparous women and those with lower socioeconomic status. It is thus necessary to strengthen preventive measures in vulnerable subgroups and maximize situations for use of health services.
\end{abstract}

Uterine Cervical Neoplasms; Vaginal Smears; Health Services Coverage; Primary Health Care
Michele da Silva Correa 1 Denise Silva da Silveira 1 Fernando Vinholes Siqueira 1 Luiz Augusto Facchini 1 Roberto Xavier Piccini 1 Elaine Thumé 1 Elaine Tomasi 1

\section{Introdução}

O câncer do colo do útero é um importante problema de saúde pública e está ranqueado como o segundo tipo de câncer mais frequente entre as mulheres no mundo. É responsável pelo óbito anual de aproximadamente 230 mil mulheres 1 sendo mais de $80 \%$ ocorridas nos países em desenvolvimento 2 .

A detecção precoce do câncer de colo uterino a partir de técnicas de rastreamento ou screening de lesões precursoras antes de se tornarem invasivas e o tratamento adequado podem prevenir o aparecimento da doença. Dentre as técnicas de detecção, a colpocitologia oncológica, teste de Papanicolaou, Pap teste ou citopatológico de colo uterino é considerado o exame mais efetivo e eficiente a ser aplicado coletivamente em programas de rastreamento 3,4,5,6,7. A Organização Mundial da Saúde (OMS) recomenda a realização do exame a cada três anos em mulheres dos 25 aos 64 anos após dois exames negativos com intervalo anual 1,2 .

Apesar de se conhecer os benefícios do exame citopatológico de colo uterino, estudos brasileiros e a revisão sistemática de Martins et al. 10, mostram que a cobertura deste exame ainda é baixa de acordo com o preconizado pela OMS que é de $80 \%$ 8,9,10,11,12,13. Com relação à adequação da periodicidade de realização de exame citopatológico, a maioria dos estudos aplica o cri- 
tério de ter realizado pelo menos um exame nos últimos três anos e tem demonstrado que esta também não alcança os indicadores desejáveis $14,15,16$. Fatores como a baixa escolaridade, baixo nível socioeconômico, ausência de filhos e não consultar com médico no último ano têm sido apontados por diversos autores como associados tanto à não realização quanto à inadequação da periodicidade do exame citopatológico 17,18,19,20 . Aspectos relacionados às crenças e atitudes da mulher em relação ao câncer de colo uterino e a autopercepção da severidade e suscetibilidade à doença também foram identificados como limitantes à realização deste exame 21,22. Características relacionadas ao serviço como a distância deste em relação ao usuário, carências de recursos materiais para a realização do exame, dificuldades no transporte e aspectos burocráticos incluindo tempo de espera tanto para marcação como para o atendimento foram mencionadas por pesquisadores como barreiras para a realização do exame 8,15,23.

Este estudo tem como objetivo analisar a cobertura e a adequação da periodicidade do exame citopatológico de colo uterino em mulheres que tiveram filho nos últimos dois anos que antecederam a realização do estudo, residentes em áreas de abrangência de unidades básicas de saúde (UBS) nas regiões Sul e Nordeste do Brasil.

\section{Métodos}

Foi realizado um estudo de delineamento transversal no ano de 2005 (Estudo de Linha de Base - ELB) com uma amostra de mulheres residentes nos estados do Rio Grande do Sul, Santa Catarina, Alagoas, Pernambuco, Paraíba, Rio Grande do Norte e Piauí, que haviam tido filho nos últimos dois anos anteriores à realização da pesquisa e residiam na área de abrangência de UBS de 41 municípios com mais de 100 mil habitantes. Os municípios incluídos no estudo compõem o Lote 2 Sul e Nordeste do ELB do Projeto de Expansão e Consolidação da Saúde da Família (PROESF) 24,25.

Uma amostra aleatória de 120 UBS foi sorteada em cada um dos lotes estudados, com distintas modalidades de atenção básica - Estratégia Saúde da Família (ESF) e tradicional. A partir de listas produzidas pelos municípios, as UBS foram selecionadas na razão de duas UBS do grupo ESF para uma UBS do grupo tradicional. A seleção da amostra nos municípios foi proporcional à capacidade instalada de sua rede básica, pareando as UBS por tamanho da área física. Na Região Sul, obteve-se uma amostra de 69 UBS de ESF e 51 tradicionais. No Nordeste, a amostra foi constituída de 79 UBS de ESF e 41 tradicionais. As UBS sorteadas orien- taram a seleção da amostra de mulheres da área de abrangência dos serviços.

A estratégia para delimitação da área de abrangência da UBS incluiu a obtenção prévia de seu mapa e uma estimativa populacional a partir das áreas censitárias do IBGE. A localização dos indivíduos na área delimitada ocorreu por meio de amostragem sistemática e incluiu apenas um indivíduo por domicílio

Os parâmetros utilizados para avaliar diferenças na cobertura populacional de ações programáticas no ELB-Universidade Federal de Pelotas (UFPel) segundo o modelo de atenção das UBS estão apresentados detalhadamente na publicação de Facchini et al. 24.

A população alvo deste estudo esteve composta por mulheres que tiveram filho nos últimos dois anos antecedentes ao início da pesquisa e residiam na área de abrangência de UBS de 41 municípios com mais de 100 mil habitantes nas regiões Sul e Nordeste do Brasil.

Todas as entrevistadas foram investigadas quanto ao conhecimento sobre o exame (não e sim) e à periodicidade recomendada para a realização do exame em cinco categorias (mais de uma vez ao ano, de ano em ano, de dois em dois anos, de três em três anos, a intervalos maiores). O desfecho dicotômico "ter realizado exame citopatológico de colo uterino na vida" foi operacionalizado através das perguntas: " $a$ senhora conhece o exame para evitar o câncer do colo do útero ou o exame de pré-câncer ou Papanicola$o u ?$ ?. Nos casos afirmativos, perguntou-se sobre a sua realização na vida e o tempo de realização do último exame em anos, meses e dias. O exame citopatológico de colo uterino foi considerado adequado quando a mulher referiu ter realizado pelo menos um exame nos últimos três anos 14 .

Para a definição dos estratos socioeconômicos foram utilizados os critérios de classificação socioeconômica do Brasil da Associação Brasileira de Empresas de Pesquisa (ABEP) 26 . O critério é um instrumento de segmentação econômica que utiliza o levantamento de características domiciliares, atribuindo pontos em função de cada característica domiciliar. Por conseguinte, é realizada a soma destes pontos e uma correspondência entre as cinco faixas de pontuação do critério e cinco estratos de classificação econômica, sendo o A o mais alto e o E o mais baixo.

No exame da consistência dos dados de variáveis dicotômicas, utilizou-se o índice kappa e para as variáveis quantitativas utilizou-se o coeficiente de correlação de Spearman. A maior parte dos itens testados apresentou índices altamente satisfatórios, acima de 0,700.

As variáveis independentes incluídas nas análises para associações foram: (a) modelo de 
atenção da UBS da área de abrangência (tradicional ou ESF); (b) idade de risco para câncer de colo uterino (até 24 anos e 25 anos e mais); (c) cor da pele (branca e parda/preta/outra); (d) escolaridade em tercis (0-5 anos, 6-8 anos, 9 anos e mais de estudo); (e) estrato socioeconômico pela $\mathrm{ABEP}$ (A, B, C, D e E); (f) primiparidade (não e sim); (g) fez pré-natal na última gestação (não e sim); (h) consultou na UBS da área de abrangência para exame ginecológico no último ano (não e sim), e (i) consultou na UBS da área de abrangência por outros motivos além do ginecológico no último ano (não e sim). As análises levaram em consideração o desenho amostral seguindo um modelo hierárquico de determinação para o desfecho. As variáveis independentes “(a)-(e)” fizeram parte do nível mais distal (Nível 1) e as “(f)-(i)" do nível proximal (Nível 2). Na análise multivariável as variáveis do Nível 2 foram ajustadas para as variáveis do mesmo nível e para as do Nível 1.

A análise inicialmente incluiu a frequência das variáveis independentes e dos desfechos através do cálculo de proporções e respectivos intervalos de 95\% de confiança (IC95\%) para toda a amostra de mulheres e estratificada de acordo com a região. A prevalência do desfecho também foi calculada para o grupo das variáveis independentes. Os valores de significância foram testados utilizando-se os testes de Wald para heterogeneidade e tendência linear. A análise ajustada foi realizada por regressão de Poisson com cálculos robustos de razões de prevalências ajustadas, IC95\% e valores de significância usando os mesmos testes descritos acima, segundo o pressuposto de que exista uma relação hierárquica entre as variáveis e o desfecho. Variáveis com valor de $\mathrm{p} \leq 0,20$ foram mantidas no modelo de análise como estratégia para controle de possível confusão. As análises foram realizadas no pacote estatístico Stata 9.2 (Stata Corp., College Station, Estados Unidos).

Os preceitos éticos da pesquisa que envolve seres humanos presentes na Resolução no. 196/96 foram observados. O estudo do PROESF foi aprovado pelo Comitê de Ética da Faculdade de Medicina da UFPel, protocolo $n^{\circ}$. 045/2004.

\section{Resultados}

A amostra deste estudo foi composta por 3.939 mulheres: 1.826 mulheres no Sul e 2.113 no Nordeste. Com a amostra final, a margem de erro para a prevalência do desfecho encontrada foi de 2,0 pontos percentuais e poder estatístico de $80 \%$. Para a análise das associações o estudo teve poder de $80 \%$ para detectar como significativas razões de prevalências de 1,5 ou maiores com nível de $95 \%$ de confiança.

A média de idade das mulheres estudadas foi de 25,7 anos ( $\mathrm{DP}=6,5)$, sendo superior no Sul $(26,4 ; \mathrm{DP}=6,9)$ quando comparada a do Nordeste $(25,1 ; \mathrm{DP}=6,1)(\mathrm{p}<0,001)$. Em relação à escolaridade a média de anos de estudos foi de 7,3 $(\mathrm{DP}=2,9)$ para toda amostra, sendo significativamente maior no Nordeste (Sul: 7,2; $\mathrm{DP}=2,9$; Nordeste: 7,4; DP = 2,9; $\mathrm{p}=0,019)$. A Tabela 1 descreve a amostra em relação às variáveis independentes estudadas por região. A proporção de mulheres da categoria idade de risco para câncer de colo uterino ( 25 anos e mais) foi de 53,9\% no Sul e $47,8 \%$ no Nordeste. Aproximadamente metade da amostra referiu ter a cor da pele branca, sendo esta prevalência significativamente maior no Sul $(71,1 \%)$ do que no Nordeste $(43,2 \%)$. O estrato socioeconômico mais prevalente foi o E $(40,8 \%)$, com predomínio desta categoria na região Nordeste $(51,9 \%)$. Quanto aos fatores reprodutivos $32,2 \%$ das mulheres eram primíparas, não havendo diferença significativa entre as regiões. Considerando a utilização de serviços de saúde no último ano, cerca de um quarto das mulheres havia realizado consulta na UBS da área de abrangência para exame ginecológico $($ Sul $=25,2 \%$; Nordeste $=23,8 \% ; p=0,307)$ e $18 \%$ consultou por outros motivos além do ginecológico $($ Sul $=19,9 \%$; Nordeste $=16,3 \% ; \mathrm{p}=0,004)$.

Na investigação sobre o conhecimento sobre o exame citopatológico de colo uterino $91,1 \%$ do total da amostra respondeu que conhecia o teste (Sul = 90,1\%; Nordeste $=91,9 \% ; p=0,059)$. Mesmo conhecendo o exame, $17,3 \%$ das mulheres relatou que nunca o havia realizado $(\mathrm{Sul}=17,6 \%$; Nordeste $=17 \% ; p=0,648$ ). Com o intuito de dimensionar esse conhecimento as mulheres foram questionadas quanto à periodicidade recomendada para a realização do exame. Para 55,7\% o exame deveria ser realizado mais de uma vez ao ano, $43,1 \%$ acreditavam que o exame deveria ser realizado anualmente e apenas $0,2 \%$ de três em três anos, sem diferenças significativas entre as regiões. Com relação ao local de realização do exame, $38,2 \%$ referiram ter realizado o último na UBS da área de abrangência de sua moradia (Sul $=39,7 \%$; Nordeste $=36,9 ; \mathrm{p}=0,122$ ).

A Tabela 2 apresenta cobertura e a adequação da periodicidade do exame citopatológico de colo uterino. A cobertura foi de 75,3\% (IC95\%: $74,0-76,7)$, não apresentando diferença significativa entre as regiões (Sul $=74,3 \%$; Nordeste = $76,2 ; \mathrm{p}=0,115)$. A prevalência de adequação da periodicidade do exame foi de 70,7 \% (IC95\%: 69,3-72,1) para total da amostra, sendo $69,4 \%$ (IC95\%: 67,3-71,6) Sul e 71,8 (IC95\%: 69,9-73,7) para Nordeste. 
Características do contexto, sociodemográficas, reprodutivas, de utilização de serviços e do conhecimento sobre o exame citopatológico de colo uterino da amostra de mulheres que tiveram filho nos últimos dois anos nas regiões Sul e Nordeste do Brasil, 2005

\begin{tabular}{|c|c|c|c|c|}
\hline Variáveis & $\begin{array}{l}\text { Total } \\
\text { n (\%) }\end{array}$ & $\begin{array}{c}\text { Sul } \\
\text { n (\%) }\end{array}$ & $\begin{array}{c}\text { Nordeste } \\
\text { n (\%) }\end{array}$ & Valor de $\mathrm{p}$ \\
\hline \multicolumn{5}{|l|}{ Contexto } \\
\hline Modelo de atenção da UBS da área de & & & & $<0,001$ \\
\hline \multicolumn{5}{|l|}{ abrangência } \\
\hline Tradicional & $1.372(34,8)$ & $744(40,7)$ & $628(29,7)$ & \\
\hline ESF & $2.567(65,2)$ & $1.082(59,3)$ & $1.485(70,3)$ & \\
\hline \multicolumn{5}{|l|}{ Demográficas } \\
\hline Idade de risco para câncer de colo uterino & & & & $<0,001$ \\
\hline Até 24 & $1.945(49,4)$ & $842(46,1)$ & $1.103(52,2)$ & \\
\hline 25 e mais & $1.993(50,6)$ & $984(53,9)$ & $1.009(47,8)$ & \\
\hline Cor da pele & & & & $<0,001$ \\
\hline Branca & $2.212(56,2)$ & $1.299(71,1)$ & $913(43,2)$ & \\
\hline Parda/Preta/Outra & $1.726(43,8)$ & $527(28,9)$ & $1.199(56,8)$ & \\
\hline \multicolumn{5}{|l|}{ Socioeconômicas } \\
\hline Escolaridade em tercis (anos) & & & & 0,004 \\
\hline $0-5$ & $1.218(30,9)$ & $593(33,9)$ & $625(32,1)$ & \\
\hline $6-8$ & $1.277(32,4)$ & $641(36,6)$ & $636(32,6)$ & \\
\hline 9 e mais & $1.203(30,4)$ & $516(29,5)$ & $687(35,3)$ & \\
\hline Estrato socioeconômico ABEP & & & & $<0,001$ \\
\hline $\mathrm{BC}$ & $1.010(26,9)$ & $646(36,7)$ & $364(18,2)$ & \\
\hline $\mathrm{D}$ & $1.216(32,3)$ & $617(35,1)$ & $599(30,0)$ & \\
\hline $\mathrm{E}$ & $1.534(40,8)$ & $497(28,2)$ & $1.037(51,9)$ & \\
\hline \multicolumn{5}{|l|}{ Reprodutivas } \\
\hline Primiparidade & & & & 0,263 \\
\hline Não & $2.670(67,8)$ & $1.253(68,7)$ & $1.417(67,1)$ & \\
\hline Sim & $1.266(32,2)$ & $570(31,3)$ & $696(32,9)$ & \\
\hline \multicolumn{5}{|l|}{ Utilização de serviços de saúde } \\
\hline Fez pré-natal na última gestação & & & & 0,831 \\
\hline Não & $97(2,5)$ & $46(2,5)$ & $51(2,4)$ & \\
\hline $\operatorname{Sim}$ & $3.840(97,5)$ & $1.779(97,5)$ & $2.061(97,6)$ & \\
\hline Consulta na UBS da área de abrangência & & & & 0,307 \\
\hline \multicolumn{5}{|l|}{ para exame ginecológico no ultimo ano } \\
\hline Não & $2.958(75,5)$ & $1.352(74,8)$ & $1.606(76,2)$ & \\
\hline Sim & $958(24,5)$ & $456(25,2)$ & $502(23,8)$ & \\
\hline Consulta na UBS da área de abrangência & & & & 0,004 \\
\hline \multicolumn{5}{|l|}{ por outros motivos além do ginecológico no } \\
\hline \multicolumn{5}{|l|}{ ultimo ano } \\
\hline Não & $3.215(82,0)$ & $1.459(80,1)$ & $1.756(83,7)$ & \\
\hline Sim & $706(18,0)$ & $363(19,9)$ & $343(16,3)$ & \\
\hline
\end{tabular}

ABEP: Associação Brasileira de Empresas de pesquisa; ESF: Estratégia Saúde da Família; UBS: unidade básica de saúde.

A Tabela 3 apresenta a associação bruta e ajustada da amostra entre as mulheres com exame citopatológico de colo uterino adequado e as variáveis independentes. Pôde-se verificar após a análise ajustada que a adequação da periodi- cidade do exame foi mais frequente entre as mulheres com 25 anos ou mais ( $R P=1,28$; IC95\%: 1,18-1,39); de maior escolaridade, especialmente entre as de nove anos ou mais de estudo $(\mathrm{RP}=$ 1,26; IC95\%: 1,13-1,39); entre as que realizaram 
Tabela 2

Cobertura e adequação da periodicidade do exame citopatológico de colo uterino da amostra de mulheres que tiveram filho nos últimos dois anos nas regiões Sul e Nordeste do Brasil, 2005.

\begin{tabular}{lcccccccc}
\hline Variável & \multicolumn{2}{c}{ Total } & \multicolumn{2}{c}{ Sul } & \multicolumn{2}{c}{ Nordeste } \\
& $\mathbf{n}$ & \% (IC95\%) & $\mathbf{n}$ & \% (IC95\%) & $\mathbf{n}$ & \% (IC95\%) & Valor de $\mathbf{p}$ \\
\hline Cobertura & 3.935 & $75,3(74,0-76,7)$ & 1.823 & $74,3(72,3-76,3)$ & 2.112 & $76,2(74,4-78,0)$ & 0,155 \\
Adequação & 2.964 & $70,7(69,3-72,1)$ & 1.796 & $69,4(67,3-71,6)$ & 2.057 & $71,8(69,9-73,7)$ & 0,107 \\
\hline
\end{tabular}

IC95\%: intervalo de 95\% de confiança.

Tabela 3

Prevalência de adequação da periodicidade do exame citopatológico de colo uterino conforme as variáveis independentes, análise bruta e ajustada da amostra de mulheres que tiveram filho nos últimos dois anos nas regiões Sul e Nordeste do Brasil, 2005.

\begin{tabular}{|c|c|c|c|c|c|}
\hline \multirow[t]{2}{*}{ Variável (nível hierárquico) * } & \multicolumn{3}{|c|}{ Análise bruta } & \multicolumn{2}{|c|}{ Análise ajustada } \\
\hline & $\%$ & RP (IC95\%) & Valor de $p$ & RP (IC95\%) & Valor de $p$ \\
\hline Modelo de atenção (1) & & & 0,849 & & 0,747 \\
\hline Tradicional & 71,1 & 1,00 & & 1,00 & \\
\hline ESF & 70,5 & $0,99(0,92-1,07)$ & & $1,01(0,93-1,10)$ & \\
\hline Idade de risco para câncer de colo uterino (1) [anos] & & & $<0,001$ & & $<0,001$ \\
\hline Até 24 & 61,9 & 1,00 & & 1,00 & \\
\hline 25 e mais & 79,2 & $1,28(1,19-1,38)$ & & $1,28(1,18-1,39)$ & \\
\hline Cor da pele (1) & & & 0,030 & & 0,886 \\
\hline Branca & 73,3 & 1,00 & & 1,00 & \\
\hline Parda/Preta/Outra & 67,3 & $0,92(0,85-0,99)$ & & $0,99(0,92-1,08)$ & \\
\hline Escolaridade (1) [anos] & & & $<0,001 * \star$ & & $<0,001 \star \star$ \\
\hline $0-5$ & 62,2 & 1,00 & & 1,00 & \\
\hline $6-8$ & 70,9 & $1,14(1,03-1,26)$ & & $1,14(1,03-1,26)$ & \\
\hline 9 e mais & 82,8 & $1,33(1,21-1,46)$ & & $1,26(1,13-1,39)$ & \\
\hline Nível socioeconômico (1) & & & $<0,001 * \star$ & & 0,006 ** \\
\hline $\mathrm{BC}$ & 81,6 & 1,00 & & 1,00 & \\
\hline$D$ & 74,6 & $0,91(0,83-1,00)$ & & $0,98(0,89-1,08)$ & \\
\hline $\mathrm{E}$ & 60,9 & $0,75(0,68-0,82)$ & & $0,86(0,78-0,96)$ & \\
\hline Primiparidade (2) & & & 0,003 & & 0,017 \\
\hline Não & 73,5 & 1,00 & & 1,00 & \\
\hline Sim & 64,8 & $0,88(0,81-0,96)$ & & $0,89(0,81-0,98)$ & \\
\hline Fez pré-natal na última gestação (2) & & & $<0,001$ & & 0,006 \\
\hline Não & 35,1 & 1,00 & & 1,00 & \\
\hline Sim & 71,6 & $2,04(1,45-2,87)$ & & $1,68(1,16-2,43)$ & \\
\hline $\begin{array}{l}\text { Consulta na UBS da área de abrangência para exame ginecológico } \\
\text { no último ano (2) }\end{array}$ & & & $<0,001$ & & $<0,001$ \\
\hline Não & 65,8 & 1,00 & & 1,00 & \\
\hline Sim & 86,1 & $1,07(1,04-1,11)$ & & $1,29(1,16-2,43)$ & \\
\hline Consulta na UBS da área de abrangência por outros motivos além & & & 0,914 & & 0,599 \\
\hline do ginecológico no último ano (2) & & & & & \\
\hline Não & 70,8 & 1,00 & & 1,00 & \\
\hline Sim & 70,4 & $0,99(0,90-1,10)$ & & $0,97(0,88-1,08)$ & \\
\hline
\end{tabular}

ESF: Estratégia Saúde da Família; IC95\%: intervalo de 95\% de confiança; RP: razão de prevalência; UBS: unidade básica de saúde.

* Na análise multivariável as variáveis do Nível 2 foram ajustadas para as variáveis do mesmo nível e para as do Nível 1 ;

** Teste de tendência linear. 
pré-natal na última gestação $(\mathrm{RP}=1,68$, IC95\%: $1,16-2,43$ ) e aquelas consultaram na UBS da área de abrangência para exame ginecológico no último ano ( $\mathrm{RP}=0,97$, IC95\%: 0,88-1,08). Por outro lado, a adequação foi menos frequente entre as mulheres de estrato socioeconômico $\mathrm{E}(\mathrm{RP}=$ 0,75; IC95\%: 0,68-0,82) e primíparas $(\mathrm{RP}=0,89$; IC95\%: 0,81-0,98).

As Tabelas 4 e 5 apresentam os resultados da análise bruta e da ajustada para as regiões Sul e Nordeste, respectivamente. Observou-se que ter 25 anos ou mais de idade e ter consultado na UBS da área de abrangência para exame ginecológico no último ano estiveram significativamente associados com adequação da periodicidade do exame citopatológico de colo uterino, em ambas as regiões. No Sul, a escolaridade também esteve associada positivamente com o desfecho. De forma contrária as mulheres do nível socioeconômico E tiveram menor frequência de adequação da periodicidade do exame nas duas regiões. No Nordeste a adequação foi menos frequente entre as primíparas. A associação entre maior adequação do citopatológico e realização de pré-natal

Tabela 4

Prevalência de adequação do exame preventivo do câncer do colo uterino conforme as variáveis independentes, análise bruta e ajustada da amostra de mulheres que tiveram filho nos últimos dois anos na Região Sul do Brasil, 2005.

\begin{tabular}{|c|c|c|c|c|c|}
\hline \multirow[t]{2}{*}{ Variável (nível hierárquico) * } & \multicolumn{3}{|c|}{ Análise bruta } & \multicolumn{2}{|c|}{ Análise ajustada } \\
\hline & $\%$ & RP (IC95\%) & Valor de p & RP (IC95\%) & Valor de p \\
\hline Modelo de atenção (1) & & & 0,757 & & 0,839 \\
\hline Tradicional & 70,2 & 1,00 & & 1,00 & \\
\hline ESF & 68,9 & $0,98(0,88-1,10)$ & & $1,01(0,90-1,14)$ & \\
\hline Idade de risco para câncer de colo uterino (1) [anos] & & & $<0,001$ & & $<0,001$ \\
\hline Até 24 & 60,5 & 1,00 & & 1,00 & \\
\hline 25 e mais & 77,1 & $1,27(1,14-1,42)$ & & $1,29(1,15-1,45)$ & \\
\hline Cor da pele (1) & & & 0,328 & & 0,780 \\
\hline Branca & 70,7 & 1,00 & & 1,00 & \\
\hline Parda/Preta & 66,4 & $0,94(0,83-1,06)$ & & $1,02(0,89-1,16)$ & \\
\hline Escolaridade (1) [anos] & & & $<0,001 \star \star$ & & $<0,001 * \star$ \\
\hline $0-5$ & 58,8 & 1,00 & & 1,00 & \\
\hline $6-8$ & 70,1 & $1,19(1,04-1,37)$ & & $1,19(1,02-1,38)$ & \\
\hline 9 e mais & 84,5 & $1,45(1,25-1,66)$ & & $1,35(1,15-1,57)$ & \\
\hline Nível socioeconômico (1) & & & $<0,001$ ** & & 0,018 ** \\
\hline $\mathrm{BC}$ & 80,5 & 1,00 & & 1,00 & \\
\hline D & 71,8 & $0,89(0,78-1,01)$ & & $0,98(0,86-1,12)$ & \\
\hline$E$ & 54,0 & $0,67(0,58-0,78)$ & & $0,80(0,68-0,95)$ & \\
\hline Primiparidade (2) & & & 0,362 & & 0,319 \\
\hline Não & 70,6 & 1,00 & & 1,00 & \\
\hline $\operatorname{Sim}$ & 66,7 & $0,94(0,84-1,07)$ & & $0,93(0,80-1,07)$ & \\
\hline Fez pré-natal na última gestação (2) & & & 0,004 & & 0,056 \\
\hline Não & 33,3 & 1,00 & & 1,00 & \\
\hline $\operatorname{Sim}$ & 70,3 & $2,11(1,27-3,51)$ & & $1,65(0,99-2,75)$ & \\
\hline $\begin{array}{l}\text { Consulta na UBS da área de abrangência para exame ginecológico } \\
\text { no último ano (2) }\end{array}$ & & & $<0,001$ & & $<0,001$ \\
\hline Não & 64,6 & 1,00 & & 1,00 & \\
\hline Sim & 84,2 & $1,30(1,15-1,50)$ & & $1,31(1,16-1,48)$ & \\
\hline $\begin{array}{l}\text { Consulta na UBS da área de abrangência por outros motivos além do } \\
\text { ginecológico no último ano (2) }\end{array}$ & & & 0,651 & & 0,947 \\
\hline Não & 69,0 & 1,00 & & 1,00 & \\
\hline Sim & 71,3 & $1,03(0,90-1,19)$ & & $0,99(0,86-1,15)$ & \\
\hline
\end{tabular}

ESF: Estratégia Saúde da Família; IC95\%: intervalo de 95\% de confiança; RP: razão de prevalência; UBS: unidade básica de saúde.

* Na análise multivariável as variáveis do Nível 2 foram ajustadas para as variáveis do mesmo nível e para as do Nível 1;

** Teste de tendência linear. 
Prevalência de adequação da periodicidade do exame citopatológico de colo uterino conforme as variáveis independentes, análise bruta e ajustada da amostra de mulheres que tiveram filho nos últimos dois anos na Região Nordeste do Brasil, 2005.

\begin{tabular}{|c|c|c|c|c|c|}
\hline \multirow[t]{2}{*}{ Variável (nível hierárquico) * } & \multicolumn{3}{|c|}{ Análise bruta } & \multicolumn{2}{|c|}{ Análise ajustada } \\
\hline & $\%$ & RP (IC95\%) & Valor de $p$ & RP (IC95\%) & Valor de $\mathrm{p}$ \\
\hline Modelo de atenção (1) & & & 0,918 & & 0,942 \\
\hline Tradicional & 72,1 & 1,00 & & 1,00 & \\
\hline ESF & 71,7 & $0,99(0,89-1,11)$ & & $1,00(0,89-1,13)$ & \\
\hline Idade de risco para câncer de colo uterino (1) [anos] & & & $<0,001$ & & $<0,001$ \\
\hline Até 24 & 63,1 & 1,00 & & 1,00 & \\
\hline 25 e mais & 81,4 & $1,29(1,16-1,42)$ & & $1,28(1,15-1,43)$ & \\
\hline Cor da pele (1) & & & 0,014 & & 0,311 \\
\hline Branca & 77,0 & 1,00 & & 1,00 & \\
\hline Parda/Preta/Outra & 67,8 & $0,88(0,79-0,97)$ & & $0,94(0,85-1,06)$ & \\
\hline Escolaridade (1) [anos] & & & $0,001 * *$ & & 0,065 ** \\
\hline $0-5$ & 65,5 & 1,00 & & 1,00 & \\
\hline $6-8$ & 71,6 & $1,09(0,95-1,25)$ & & $1,08(0,94-1,25)$ & \\
\hline 9 e mais & 81,5 & $1,24(1,09-1,41)$ & & $1,15(0,99-1,33)$ & \\
\hline Nível socioeconômico (1) & & & $<0,001$ ** & & 0,045 ** \\
\hline $\mathrm{BC}$ & 83,6 & 1,00 & & 1,00 & \\
\hline $\mathrm{D}$ & 77,5 & $0,92(0,80-1,07)$ & & $0,97(0,84-1,13)$ & \\
\hline $\mathrm{E}$ & 64,3 & $0,77(0,67-0,88)$ & & $0,86(0,74-1,01)$ & \\
\hline Primiparidade (2) & & & $<0,001$ & & 0,017 \\
\hline Não & 76,1 & 1,00 & & 1,00 & \\
\hline Sim & 63,3 & $0,83(0,74-0,93)$ & & $0,86(0,76-0,97)$ & \\
\hline Fez pré-natal na última gestação (2) & & & 0,004 & & 0,059 \\
\hline Não & 36,7 & 1,00 & & 1,00 & \\
\hline Sim & 72,7 & $1,98(1,24-3,14)$ & & $1,66(0,98-2,82)$ & \\
\hline $\begin{array}{l}\text { Consulta na UBS da área de abrangência para exame ginecológico no } \\
\text { último ano (2) }\end{array}$ & & & $<0,001$ & & $<0,001$ \\
\hline Não & 66,8 & 1,00 & & 1,00 & \\
\hline $\operatorname{Sim}$ & 87,8 & $1,31(1,17-1,47)$ & & $1,28(1,14-1,45)$ & \\
\hline $\begin{array}{l}\text { Consulta na UBS da área de abrangência por outros motivos além do } \\
\text { ginecológico no último ano (2) }\end{array}$ & & & 0,584 & & 0,580 \\
\hline Não & 72,2 & 1,00 & & 1,00 & \\
\hline Sim & 69,5 & $0,96(0,83-1,10)$ & & $0,96(0,83-1,11)$ & \\
\hline
\end{tabular}

ESF: Estratégia Saúde da Família; IC95\%: intervalo de 95\% de confiança; RP: razão de prevalência; UBS: unidade básica de saúde.

* Na análise multivariável as variáveis do Nível 2 foram ajustadas para as variáveis do mesmo nível e para as do Nível 1 ;

** Teste de tendência linear.

na última gestação ficou no limiar de significância nas duas regiões.

\section{Discussão}

Realizar periodicamente o exame citopatológico é a estratégia mais adotada para o rastreamento do câncer de colo uterino. A alta cobertura da população alvo é o componente mais importante no âmbito da atenção primária à saúde para a re- dução da incidência e da mortalidade por câncer do colo do útero 27 .

O presente estudo revelou que mais de $90 \%$ das mulheres estudadas conheciam o exame citopatológico de colo uterino, contudo para $99 \%$ destas mulheres constatou-se conhecimento inadequado quanto à periodicidade de realização do exame, caracterizando um achado semelhante ao encontrado em outros estudos 8,21,23,28. Destaca-se que apesar do amplo conhecimento sobre o exame, para a maioria das mulheres ele 
deve ser realizado mais de uma vez por ano ou anualmente, o que levaria a um aumento dos custos sem redução significativa nos potenciais benefícios 8,9,12,17.

A cobertura do exame citopatológico encontrada foi de $75 \%$ nas mulheres residentes na área de abrangência de UBS. Este achado é superior ao encontrado em outros estudos 13,15,16,29 e inferior ao parâmetro recomendado pela OMS para o efetivo controle do câncer de colo uterino, que é no mínimo de $80 \% 30$.

Quanto à adequação observou-se que apenas $71 \%$ das mulheres realizaram o exame com periodicidade adequada, ou seja, um exame nos últimos três anos. Tal achado é menor que o encontrado em estudos anteriores 10,16,28,31,32.

A idade das mulheres com maior prevalência de citopatológico adequado foi de 25 anos ou mais, configurando-se como um fato positivo, devido à recomendação nacional que preconiza a idade para início da coleta do exame a partir dos 25 anos 1 .

Assim como em outros estudos as mulheres com maior escolaridade e de maior nível socioeconômico tiveram maior chance de ter citopatológico adequado quando comparadas àquelas de menor escolaridade e menor nível socioeconômico 11,33. Sabendo que menores escolaridade e nível socioeconômico são fatores de risco para câncer do colo uterino 9,11,13,17,33 este estudo encontrou que as mulheres mais vulneráveis foram exatamente as que menos realizaram o exame.

As mulheres com mais de um filho e que realizaram exame ginecológico no último ano tiveram maior probabilidade de ter a periodicidade do exame adequado. Resultado semelhante a este foi observado em um estudo no qual ter filhos e consultar com médico no último ano foram os fatores associados mais importantes para a realização do exame citopatológico. No estudo os autores sugeriram que a prática do exame associada à maternidade além de aumentar a possibilidade de acesso ao serviço durante a gestação a mulher sente necessidade adicionais de auto cuidado e prevenção 34 .

Considerando os aspectos metodológicos desta pesquisa é importante salientar que as mulheres incluídas no estudo podem apresentar maior possibilidade de estarem com exame citopatológico atualizado devido à condição de grávidas nos últimos dois anos anteriores à pesquisa, o que demanda maior utilização de serviços para realização do pré-natal, revisão de parto e atendimento de intercorrências. Esta condição pode levar naturalmente a uma maior cobertura do exame preventivo, ou até uma superestimativa do resultado 35 . No entanto, os resultados encontrados não atingiram os níveis de efetividade preconizados pela OMS, evidenciando que em boa medida a chance de fazer o rastreamento durante o pré-natal não está sendo aproveitada.

Por fim, concluímos que este estudo atinge seu objetivo de descrever a cobertura e adequação da periodicidade do exame citopatológico do colo uterino, em duas importantes regiões do Brasil. Seus resultados indicam que ainda é necessário ampliar e fortalecer as ações preventivas ofertadas pelos serviços de saúde, especialmente para subgrupos de mulheres mais vulneráveis potencializar as situações que demandam utilização dos serviços de saúde, assim como realizar outros estudos com diferentes delineamentos que contribuam para compreender como estão sendo seguidas as recomendações com base no exame citopatológico do colo uterino. 


\section{Resumo}

O câncer de colo do útero é o segundo mais comum em mulheres no Brasil e no mundo e pode ser evitado através da detecção precoce de lesões precursoras. O exame citopatológico de colo uterino ainda é o mais efetivo e eficiente para realizar este rastreamento. O objetivo do estudo foi investigar a cobertura e a adequação do exame citopatológico e fatores associados. Foi realizado um estudo transversal com 3.939 mulheres que tiveram filho nos últimos dois anos anteriores à pesquisa, distribuídas em 41 municípios brasileiros. A cobertura do exame na vida foi de 75,3\% (IC95\%: 74,0-76,7) e a adequação foi de 70,7\% (IC95\%: 69,3-72,1). A adequação associou-se positivamente com idade maior de 25 anos, maior escolaridade, fazer pré-natal na última gestação e consultar para exame ginecológico no último ano. Foi menos frequente entre mulheres do estrato socioeconômico mais baixo e primíparas. Portanto, faz-se necessário fortalecer as ações preventivas para os subgrupos de mulheres mais vulneráveis, assim como potencializar as situações de utilização dos serviços de saúde.

Neoplasias do Colo do Útero; Esfregaço Vaginal; Cobertura de Serviços de Saúde; Atenção Primária à Saúde

\section{Referências}

1. Instituto Nacional de Câncer. Estimativa 2010: incidência de câncer no Brasil. Rio de Janeiro: Instituto Nacional de Câncer; 2011.

2. World Health Organization. Comprehensive cervical cancer control: a guide to essential practice. Geneva: World Health Organization; 2007.

3. Pinho AA, França-Junior I. Prevenção de câncer de colo do útero: um modelo teórico para analisar o acesso e a utilização de teste de Papanicolau. Rev Bras Saúde Matern Infant 2003; 3:95-112.

4. Castro-Jiménez MA, Vera-Cala LM, Posso-Valencia HJ. Epidemiología del cáncer de cuello uterino: estado del arte. Rev Colomb Obstet Ginecol 2006; $57: 182-9$.

\section{Colaboradores}

M. S. Corrêa, D. S. Silveira e F. V. Siqueira participaram da concepção do artigo, análise de dados, interpretação e redação final do artigo. L. A. Facchini, R. X. Piccini, E. Tomasi e E. Thumé participaram na revisão crítica e redação final do manuscrito.

\section{Agradecimentos}

Os autores agradecem aos gestores, coordenadores de atenção básica à saúde e do Programa Saúde da Família, e aos trabalhadores das unidades básicas da saúde o apoio prestado à realização do estudo. Este trabalho contou com o apoio financeiro da Coordenação de Aperfeiçoamento de Pessoal de Nível Superior (Capes), Ministério da Saúde e Banco Mundial e integrou o Componente 3 do Projeto de Expansão e Consolidação Saúde da Família (PROESF).
5. Oliveira MM, Pinto IC. Percepção das usuárias sobre as ações de prevenção do câncer do colo do útero na Estratégia Saúde da Família em uma distrital de saúde do município de Ribeirão Preto, São Paulo, Brasil. Rev Bras Saúde Matern Infant 2007; 7:31-8.

6. Salomon D, Breen N, McNeel T. Cervical cancer screening rates in the United States and the potential impact of implementation of screening guidelines. CA Cancer J Clin 2007; 57:105-11.

7. Moscicki AB. HPV Vaccines: today and in the future. J Adolesc Health 2008; 43:26-40. 
8. Pinho AA, França Junior I, Schraiber LB, D'Oliveira AFPL. Cobertura e motivos para a realização ou não do teste de Papanicolaou no Município de São Paulo. Cad Saúde Pública 2003; 19 Suppl 2:S303-13.

9. Quadros CAT, Victora CG, Costa JSD. Coverage and focus of a cervical cancer prevention program in southern Brazil. Rev Panam Salud Pública 2004; 16:223-32.

10. Martins LFL, Thuler LCS, Valente JG. Cobertura do exame Papanicolau no Brasil e seus determinantes: uma revisão sistematizada da literatura. Rev Bras Ginecol Obstet 2005; 27:485-92.

11. Oliveira MMHN, Silva AAM, Brito LMO, Coimbra LC. Cobertura e fatores associados à não realização do exame preventivo de Papanicolau em São Luís, Maranhão. Rev Bras Epidemiol 2006; 9 : 325-34.

12. Rodrigues Neto JFR, Figueiredo MFS, Siqueira LG. Exame citopatológico do colo do útero: fatores associados a não realização em ESF. Rev Eletrônica Enferm 2008; 10:610-21.

13. Albuquerque KM, Frias PG, Andrade CLTA, Aquino EML, Menezes G, Szwarcwald CL. Cobertura do teste de Papanicolaou e fatores associados à nãorealização: um olhar sobre o Programa de Prevenção do Câncer do Colo do Útero em Pernambuco, Brasil. Cad Saúde Pública 2009; 25 Suppl 2:S301-9.

14. Day NE. The epidemiological basis for evaluation of different screening policies. In: Hakama M, Miller AB, Day NE, editors. Screening for cancer of the uterine cervix. Lyon: International Agency for Research on Cancer; 1986. p. 149-60. (IARC Scientific Publications, 76).

15. Dias-da-Costa JS, Olinto MTA, Gigante DP, Menezes AMB, Macedo S, Borba AT, et al. Cobertura do exame citopatológico na cidade de Pelotas, Rio Grande do Sul, Brasil. Cad Saúde Pública 2003; 19:191-7.

16. Leal MC, Gama SGN, Frias P, Szwarcwald CL. Healthy lifestyles and access to periodic health exams among Brazilian women. Cad Saúde Pública 2005; 21 Suppl 1:S78-88.

17. Hackenhaar AA, Cesar JA, Domingues MR. Exame citopatológico de colo uterino em mulheres com idade entre 20 e 59 anos em Pelotas, RS: prevalência, foco e fatores associados à sua não realização. Rev Bras Epidemiol 2006; 9:103-11.

18. Amorin VMSL, Barros MBA, César CLG, Carandina L, Goldbaum M. Fatores associados à não realização do exame de Papanicolaou: um estudo de base populacional no Município de Campinas, São Paulo, Brasil. Cad Saúde Pública 2006; 22:2329-38.

19. Cruz LMB, Loureiro RP. Comunicação na abordagem preventiva do câncer do colo do útero: importância das influências histórico-culturais e da sexualidade feminina na adesão às campanhas. Saúde Soc 2008; 17:120-31.

20. Cirino FMSB, Nichiata LYI, Borges ALV. Conhecimento, atitude e práticas na prevenção do câncer. Esc Anna Nery Rev Enferm 2010; 14:126-34.

21. Pinho AA, França-Junior I. Prevenção do câncer de colo do útero: um modelo teórico para analisar o acesso e a utilização do teste de Papanicolaou. Rev Bras Saúde Matern Infant 2003; 3:95-112.
22. Brito CMSB, Nery IS, Torres LC. Sentimentos e expectativas das mulheres acerca da Citologia Oncótica. Rev Bras Enferm 2007; 60:387-90.

23. Gonçalves CL, Duarte G, Dias-da-Costa JS, Quintana SM, Marcolin AC. Perdas de oportunidades na prevenção do câncer de colo uterino durante o pré-natal. Ciênc Saúde Coletiva 2011; 16:2501-510.

24. Facchini LA, Piccini RX, Tomasi E, Thumé E, Teixeira VA, Silveira DS, et al. Avaliação de efetividade da Atenção Básica à Saúde em municípios das regiões Sul e Nordeste do Brasil: contribuições metodológicas. Cad Saúde Pública 2008; 24 Suppl 1:S159-72.

25. Facchini LA, Piccini RX, Tomasi E, Thumé E, Silveira DS, Siqueira FV, et al. Desempenho do PSF no sul e no nordeste do Brasil: avaliação institucional e epidemiológica da atenção básica à saúde. Ciênc Saúde Coletiva 2006; 11:669-81.

26. Associação Brasileira de Empresas de Pesquisas. Critério de classificação econômica Brasil. http:// www.abep.org/codigosguias/ABEP_CCEB.pdf (acessado em 12/Nov/2011).

27. World Health Organization. Programmes and projects. Cancer. Screening and early detection of cancer. http://www.who.int/cancer/detection/ cytologyscreen/en/index.htm (acessado em 08/ Fev/2011).

28. Fernandes RAQ, Narchi NZ. Conhecimento de gestantes de uma comunidade carente sobre os exames de detecção precoce do câncer cérvico-uterino e de mama. Rev Bras Cancerol 2002; 48:223-30.

29. Gakidou E, Nordhagen S, Obermeyer Z. Coverage of cervical cancer screening in 57 countries: Low average levels and large inequalities. PLoS Med 2008; 5:863-8.

30. World Health Organization. World cancer report 2008. Geneva: World Health Organization; 2008.

31. Szwarcwald CL, Mendonça MHM, Andrade CLT. Indicadores de atenção básica em quatro municípios do Estado do Rio de Janeiro, 2005: resultados de inquérito domiciliar de base populacional. Ciênc Saúde Coletiva 2006; 11:643-55.

32. Bottari CMS, Vasconcellos MM, Mendonça MHM. Câncer cérvico-uterino como condição marcadora: uma proposta de avaliação da atenção básica. Cad Saúde Pública 2008; 24 Suppl 1:S111-22.

33. Cesar JA, Horta BL, Gomes G, Houlthausen RS, Willrich RM, Kaercher A, et al. Fatores associados à não realização de exame citopatológico de colo uterino no extremo sul do Brasil. Cad Saúde Pública $2003 ; 19: 1365-72$.

34. Novaes HMD, Braga PE, Schout D. Fatores associados à realização de exames preventivos para câncer nas mulheres brasileiras, PNAD 2003. Ciênc Saúde Coletiva 2006; 11:1023-35.

35. Gonçalves CV, Duarte G, Costa JSD, Quintana SM, Marcolin AC. Perdas de oportunidades na prevenção do câncer de colo uterino durante o pré-natal. Ciênc Saúde Coletiva 2011; 16:2501-10.

Recebido em 09/Mai/2012

Versão final reapresentada em 26/Jul/2012 Aprovado em 17/Ago/2012 